\title{
What to do in an oncology department to face the new COVID-19 era challenges?
}

\author{
S. K. Garattini ${ }^{1,3}$ (D) A. Bin ${ }^{1} \cdot$ R. Donato ${ }^{1} \cdot$ M. Mansutti ${ }^{1} \cdot$ S. Rizzato ${ }^{1} \cdot$ G. Troiero ${ }^{1} \cdot$ A. Candoni ${ }^{2} \cdot$ R. Fanin ${ }^{2} \cdot$ G. Fasola $^{1}$
}

Received: 11 April 2020 / Accepted: 20 July 2020 / Published online: 29 July 2020

○) Springer Science+Business Media, LLC, part of Springer Nature 2020

\begin{abstract}
Italy was the first European country to be hit by COVID-19 pandemic. As a consequence, Italian oncologists had to guarantee essential treatments although minimizing exposure to the virus, and accidental infection, of patients and healthcare professionals. As Department of Medical Oncology of the University Hospital of Udine, in this short report, we describe the measures that we have taken, and gradually updated, since February 26, 2020. All accesses to our Oncology facilities are currently regulated by entrance check-points where patients are screened for infections following dedicated algorithms. Up to date, after 6 weeks of systematic execution of swabs no physician, nurse or other individual of the staff has been found positive to COVID-19. Only one patient admitted for therapy has been identified as COVID-19 positive. The aim of our work is to propose a model, made up of a set of operative procedures, that may be adopted by all the oncologists that daily struggle to guarantee safety and care in Oncology during this COVID-19 emergency.
\end{abstract}

Keywords Medical oncology $\cdot$ COVID-19 $\cdot$ Medical management $\cdot$ Pandemic

\section{Introduction}

The city of Wuhan, in China, was hit by a novel coronavirus called SARS-CoV2 by December 2019, causing a novel acute respiratory disease known as COVID-19 [1]. Soon it was made clear that the possible origin of this virus could be the seafood and animal market of the town, in Hubei province, China [2,3]. As a consequence of this outbreak, an aggressive policy of isolation of individuals was pursued by the Chinese Government. Soon it was clear that COVID-19

S. K. Garattini

silvioken@hotmail.it

A. Bin

alessandra.bin@asufc.sanita.fvg.it

R. Donato

raffaela.donato@asufc.sanita.fvg.it

M. Mansutti

mauro.mansutti@asufc.sanita.fvg.it

S. Rizzato

simona.rizzato@asufc.sanita.fvg.it

G. Troiero

graziella.troiero@asufc.sanita.fvg.it

A. Candoni

anna.candoni@asufc.sanita.fvg.it would have become a Pandemic as it reached the status of a new disease spreading rapidly worldwide in a sustained fashion (declaration of pandemic by the World Health Organization was released on March 11, 2020). Italy was recognized to be the first country in Europe to face an outbreak of the virus, and it was also the first European country to put into practice measures of "social distancing" and "home quarantine" of affected patients first until the moment when it became obvious that the same restrictions were to be applied to every Italian citizen so that by the 9th of March the Italian

\footnotetext{
R. Fanin

renato.fanin@asufc.sanita.fvg.it

G. Fasola

gianpiero.fasola@asufc.sanita.fvg.it

1 Department of Oncology, ASUFC University Hospital of Udine, 33100 Udine, UD, Italy

2 Department of Specialized Medicine, Haematolgy Clinic, ASUFC University Hospital of Udine, 33100 Udine, UD, Italy

3 Oncology Department, Azienda Sanitaria Universitaria Integrata Di Udine, Piazzale Santa Maria della Misericordia, 15, 33100 Udine, UD, Italy
} 
Prime Minister, in accordance with an emergency scientific task force, decided to "lockdown" the entire Country. On the field of Public Healthcare, Italy faced an unprecedented need to reconvert almost all elective clinical activities (i.e., non-urgent surgery and programmed ambulatory visits) into emergency and intensive care unit medical practice. To the date of 7th April, Italy registered 135,586 total cases of COVID-19, 94,067 positive individuals and more than 17,127 deaths, as reported by the Italian "Istituto Superiore di Sanità" [4]. Italian medical Oncologists were among the first professionals to decide how to guarantee essential treatments thus minimizing exposure and accidental infection of patients and healthcare professionals by SARS-CoV2 $[5,6]$. In fact, oncology patients represent a particularly vulnerable category exposed to immune depression often caused by anticancer treatment as well as by the underling disease [7]. On the one hand, this represents the greatest risk factor for fatal outcome by the infection operated by this SARS Coronavirus but, on the other, healthcare professionals involved in cancer care could be easily exposed to this chain of transmission due to, both, infections propagated by vulnerable patients and continuous incubation made by asymptomatic physician and nurses from oncology. Recently, the Italian Association for Medical Oncology (AIOM) has produced recommendation about how to cope with oncology clinical activity during the persistent emergency caused by COVID19, followed by the European Society for Medical Oncology [5]. It is thus important to somehow prioritize processes in oncology [8]. A group of young Italian oncologists have proposed some general procedures to manage oncology activity in the era of this pandemic differentiating three main settings: follow-up management, active treatment decisions and hospital admission workup [7]. As Department of Oncology of the University Hospital of Udine, in the North-Eastern Italian Region of Friuli Venezia Giulia, we manage about 1600 first consultations and 10,000 drug administration sessions per year, 50-60 patients who daily access Day Hospital (DH, the facility dedicated to cancer treatment sessions) and 40-50 other accessing to Outpatient Clinics (facility where first visits and re-assessments take place). Our group was actively involved, by the very beginning of this emergency, in designing local procedures integrating the indications made by the Italian and regional Government. Indeed, our Hub Academic Hospital serves most of the cancer care of a region accounting for about 1,200,000 inhabitants.

The Friuli Venezia Giulia was, as well, one of the first Italian Regions to adopt the most stringent rules for containing the spread of the viral outbreak started in the near Lombardia and Veneto.

In this short report, we describe the measures that we have taken, and gradually updated, since February 26, 2020. We also give a report of the first results regarding patients and staff surveillance.
In order to ensure the continuum of care and organize the protective measures needed for COVID-19, four main questions have to be answered: how to provide access and protection to patients, how to re-organize the activities, how to protect healthcare workers, how to manage the ongoing clinical trials.

\section{Access to the facilities and patient protection}

\section{Day hospital}

- All the patients accessing the DH must be negative to a structured triage carried out by trained staff (nurse or social assistance operator) through an interview, a selfcertification module (attached document 1) and the body temperature detection. In case of doubtful or positive triage, an assessment by the doctor on duty is required. The access follows the procedures shared with the Hematology Department, due to some common spaces of DH (reception area, waiting room): see Algorithm 1 (attached document 1). Patients resulting negative from the triage proceed to the $\mathrm{DH}$ wearing surgical mask, following an accurate hand washing and keeping social distance.

- To guarantee the minimal possible amount of individuals accessing the DH, the companions' access is not allowed (only exception for non-autonomous patients). The administration area, the blood-drawing area and the outside waiting rooms are forbidden to the companions. In the event the access of a companion is needed $(\max 1$ person), he/she must fill out the self-certification module and must be negative to the triage; to access the $\mathrm{DH}$ it is compulsory to wear the surgical mask and carry out hands' hygiene.

- In case of positive triage, the oncologist on duty follows the procedures indicated in Algorithm 2.

- The oncologists operating the treatment visits assess the biological characteristics of the tumor, patient's clinical scenario, the prognosis in relation to potential health risks from SARS-CoV2 infection and define the cases for which it is possible to postpone the treatment or remodulate its intensity. The following accesses are rescheduled (when necessary, by sharing the case with the reference medical doctor). In the case of re-scheduling in absence of the patient, the latter will be notified by the executive medical doctors or by the DH secretary.

- Clinical information about DH patients to previously authorized relatives are mainly provided by telephone or, in selected cases, in a fashion like the one used in the Ward Area (using external spaces). 


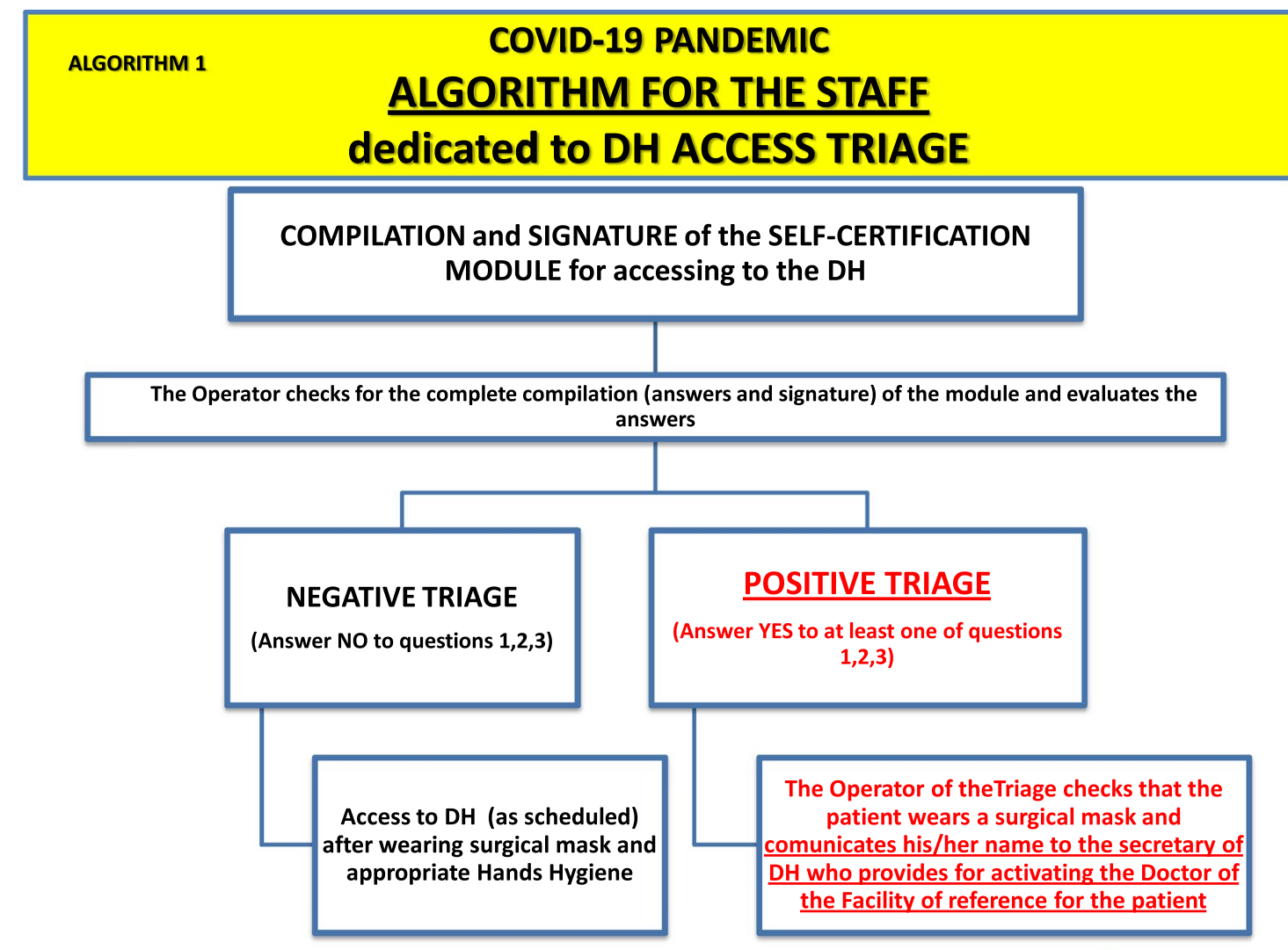

WARNING: WITH POSITIVE TRIAGE put the patient SURGICAL MASK and: 1)IF No symptoms or mild SYMPTOMS $\rightarrow$ wait OUTSIDE THE DH; b) Only IF NON-MILD SYMPTOMS $\Rightarrow$ SEAT HIM in the DH DEDICATED WAITING ROOM.

Algorithm 1 Instruction for DH triage. The nurse or social assistance operator standing outside the $\mathrm{DH}$ is dedicated to the triage. This staff follows the conduct described in the diagram after administer-

\section{Outpatient clinics}

- All the patients accessing the Outpatient Clinics must result negative to the structured triage carried out by the secretary staff (behind a glass barrier) thorough an interview and a self-certification module, following shared procedures (Algorithms 1 and 2). In case of a positive triage, the oncologist on duty assesses the case following the procedures in Algorithm 2. In the case of doubtful triage, the secretary staff requests assessment by an oncologist. Patients with negative triage proceed to the Clinics wearing a surgical mask and following an accurate hand washing.

- To guarantee the minimal possible amount of individuals entering the Clinics, the access of companions is not allowed (only exception for non-autonomous patients) to secretary staff area, to external waiting rooms and to the clinics as well. In the event the access of a companion is needed (max 1 person), he/she must fill out the self-certification module and must be negative to the ing the self-certification module (attachment 1) and after evaluating the answers. The algorithm provides the measures to be put into place upon a negative or positive triage triage procedure; to access the Clinics, it is compulsory to wear the surgical mask and carry out hands' hygiene.

- Follow-up visits (i.e., visit of patients cured from cancer) are suspended, while the activity concerning first visits, priority visits and reassessment visits are operating, except for some cases at the discretion of the executive medical doctors (see "reorganization of clinic activities" chapter).

\section{Ward area}

- All the patients accessing the integrated oncology-radiotherapy and palliative care Ward upon a programmed admission must perform a SARS-CoV2 swab within 24-48 $\mathrm{h}$ preceding the hospitalization and it must result negative before the patient is admitted.

- Specifically, in case of programmed admission for therapy, a SARS-CoV2 swab and a pre-therapy blood draw are performed by a dedicated nurse (wearing adequate personal protection), in $\mathrm{DH}$, followed by clinical assess- 


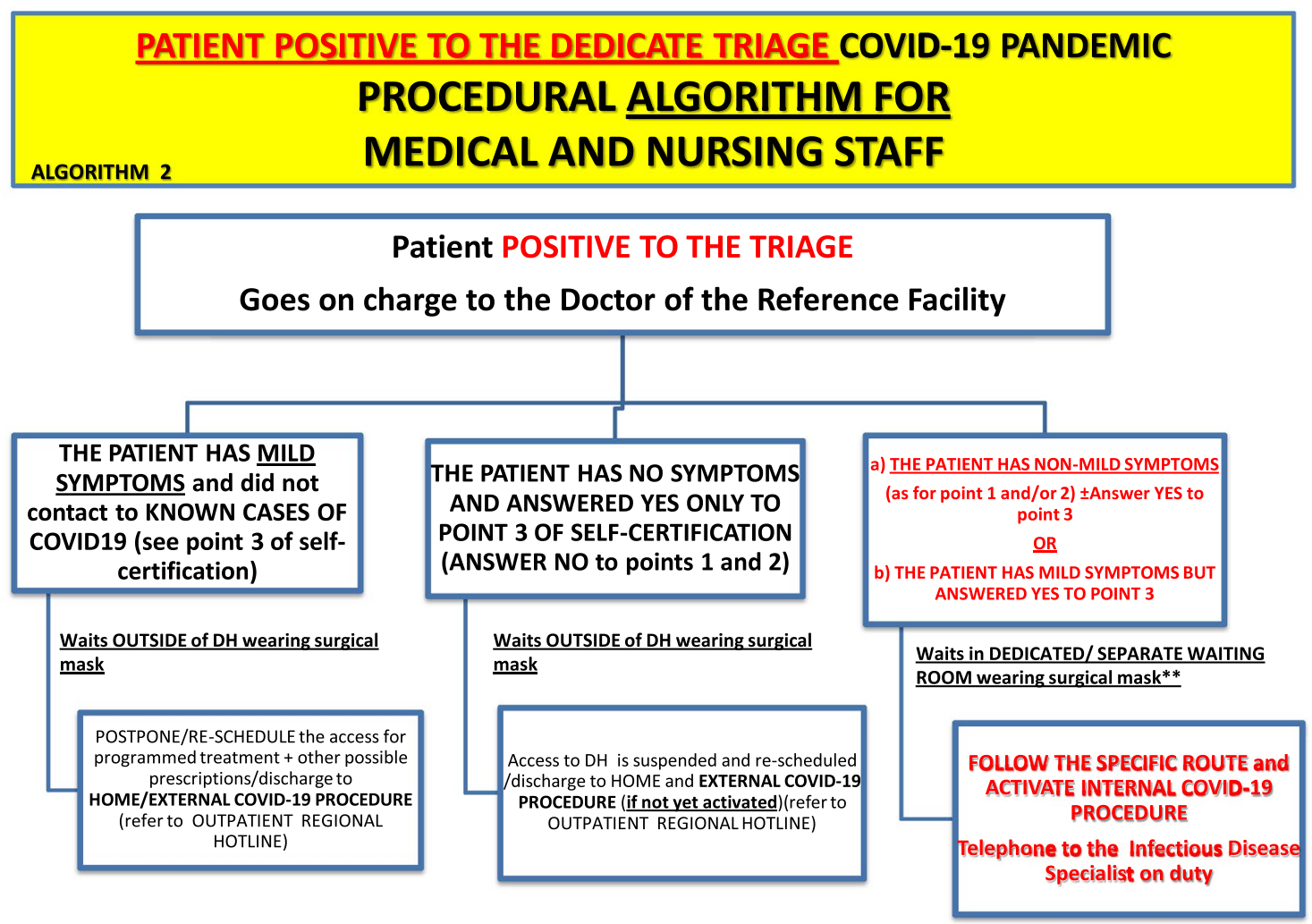

WARNING: ** IF multiple TRIAGE POSITIVE patients attend the DEDICATED WAITING ROOM, check for the respect of reciprocal security distance.

Algorithm 2 Instruction for medical and nursing staff upon positive triage. In case of positive triage (see attachment 1), the staff follows the conduct described in this diagram. Three main scenarios and their management are represented: patient with mild symptoms without

ment by the medical doctor on duty who produces a "Visit for therapy" and fills out the workup. The dedicated room lies isolated from the rest of DH. The patient returns then back home. The exams and swab results are assessed form the oncologist of the Ward, who makes a telephone call to the patient and confirms/postpones the hospitalization, validates the therapy and shares the decision with the nurse in the hospitalization area.

- The patient transferred to the Ward from another facility (or form the Emergency ER) or from another hospital can access only in case of recent negative swab (within $\max 48 \mathrm{~h}$ ) to be executed even in the absence of specific symptoms.

- The patient discharged to external Facilities (e.g., Nursing home, Residences, Hospice, or other Hospitals) must result negative to the swab within the $48 \mathrm{~h}$ preceding the release.

- The repetition of swabs for recovered and isolated patients are carried out following the Clinical Risk Management Unit procedures or at the discretion of the consultant oncologist responsible for the Ward, to patients known contact to COVID-19-positive subjects, patient with no symptom but had known contact with COVID-19-positive subject, patient with non-mild symptoms or mild symptoms with known contact with COVID-19-positive subject

with COVID-19 suspicious symptoms and/or in case of the presence of positive swabs from other patients and/ or from the staff operating in the Ward.

- As a rule, visits from relatives are not allowed, except for cases regulated by the charge nurse or by the medical doctor responsible for the Ward, after the assessment of the specific case. The authorized patients must not show any respiratory symptom, wear the surgical mask and carry out hands' hygiene. Relatives with respiratory symptoms are not allowed for visits in any case.

- Information for families (or other care-givers) authorized by the patient are provided by the oncologist by telephone call. In the case "in-person conversation" is clearly needed, it must be carried out outside the Ward, preferably with one person only; both the medical doctor who provides information and the interlocutor must wear the surgical mask.

- Students, medical residents, voluntary organization representatives and other attendees are not allowed.

- All the inquiries and non-essential or non-urgent procedures for the therapy programs that involve the transfer 
of the patient outside the ward must be reconsidered and possibly postponed; if confirmed, the patient will exit the Ward wearing the surgical mask.

\section{Reorganization of clinical activities}

- Follow-up visits: Suspended. The Secretary staff notifies to patients with programmed follow-up visits not to attend them in the hospital and informs that they will be called by telephone by the doctor, normally on the same day the visit was scheduled. The secretary accepts patients on the scheduled day with the "remote visit" provision. At the end of the telephone interview, the treatment report is produced and sent to the patient, together with the referrals (prescriptions) for the following exams: the documentation sent by e-mail should be zipped and encrypted when possible or sent by mail service (by the Secretary) when necessary. This procedure is applicable to patients affected by the most prevalent cancer types that are usually cured with surgery or after adjuvant chemotherapy (i.e., mostly breast cancers, colorectal cancers and other gastro-intestinal cancers). Visits are otherwise shortly reprogrammed in case of symptoms or examinations that may result suspect for recurrence of disease after the telephone consultation.

- First consultations, priority and reassessment visits: regularly going on, except for visits deemed deferrable by the executive oncologist (e.g., reassessment visit with stable disease on radiological report and stable clinical condition or first consultation when histological exam is not yet available).

- Therapy visits: to limit accesses, the oncologists assess the biological characteristics of the tumor, patient's clinical scenario, the prognosis in relation to potential health risks from COVID-19 infection and define the cases for which it is possible to postpone the treatment or re-modulate its intensity. Re-scheduling is especially considered for patient in advanced treatment lines for which very limited efficacy is expected by the treatment. Maximal priority is instead guaranteed for treatments of proven high probability of efficacy or for adjuvant treatments in a curative setting. The following accesses are re-scheduled by short, medium or long term according to the necessity (when needed, by sharing the case with the reference oncologist). Reference oncologist, regardless the shifts, re-examine whenever possible the program of the patients under their responsibility, canceling or postponing, whenever possible, the unnecessary visits.

- Multidisciplinary activities and facility clinical meeting: suspended. Possible clinical situations requiring multidisciplinary discussion are debated among medical doctors from different Facilities by telephone or by physical meeting only in case rules of social safety distance and protection equipment adequacy are respected. Multiprofessional meetings are held in Ward area with these measures.

- Consultancies: whenever possible and for limited contexts, the Consulting activities provided by Department of Oncology or medical advices requested by other Units are fulfilled by telephone or by remote to limit the movements/flows of patients and medical personnel within different assistance facilities.

- Urgent visits and non-programmed accesses: regularly continuing. Patients with issues related to treatment toxicities or to the management of severe symptoms related to cancer are included in this category. Patients are submitted to triage and body temperature detection before accessing. In the event of doubtful or suspicious cases, Algorithm 2 procedures are triggered.

\section{Personnel protection}

- All medical doctors and personnel operating in the Oncology Department are submitted, starting from 18.3.20 to SARS-CoV2 basal swab and periodically reassessed (every week for two weeks, then every 2 weeks).

- All medical doctors and personnel operating in the Oncology Department who refer temperature or symptoms associated with infection should promptly communicate it to the Director of the Department and then be evaluated by the infectious risk management Hospital task force.

- Medical doctors visit with surgical mask and disposable gloves. Additional protections are adopted according to case by case risk assessment, as indicated by Risk Management Unit of our Hospital.

- Each medical doctor and operator in the area takes care of his/her own workplace and integrates the daily sanitization (already foreseen) in the common workplace areas. Daily work uniform change is recommended to each medical doctor and operator.

\section{Research activities}

The suspension of recruitment activities is recommended, except for selected interventional studies (potential benefit assessed case by case). The monitoring of clinical studies, when non-deferrable, must be carried out by remote. For the patients already on treatment within the scope of clinical study, it is recommendable to substitute the activities foreseen by procedures, whenever possible, with contact by telephone (e.g., lifestyle surveys, safety visits). 


\section{Other indications}

- Avoid gatherings as far as possible. It is necessary to maintain the social distancing and avoid contacts with other operators.

- Except for "doctor on call duties" and strict necessities, avoid as far as possible interactions among people from different teams (ward team, clinic team, DH team).

- Keep tidy and clean (sanitizing) workplaces before and after every use (including mouse, keyboards, telephone handset, handles, etc.).

- Frequently aerate, whenever possible, used premises.

- As usual, it is recommended not to wear necklaces nor jewelry.

- Possible not-deferrable meetings must be carried out with the strictly needed amount of people in premises compatibles with social distancing.

Up to date, after 6 weeks of systematic execution of swabs (one test for each operator, every week for two weeks for all the 91 people daily operating in our Oncology), no physician, nurse or other individual of the staff has been found positive to SARS-CoV2 in the three articulations of our Department (after 1116 total swabs). Only one patient admitted for therapy has been identified SARS-CoV2 positive, but 9 days after accessing and in a home setting; the controls on contacts with the health personnel resulted negative. From March 6, 2020, swabs are performed to every patient entering $\mathrm{DH}$ for a therapeutic infusion at least $30 \mathrm{~min}$ long.

In conclusion, we strongly believe that all these prudential measures, which have been adopted since February 26, 2020 and gradually updated in our Oncology Department, together with the Hematology Department, could serve as a model to be replicated. In the perspective of the next months, it will be crucial to maintain this same prudential attitude towards prevention of infection by SARS-CoV2, even after the re-opening of social activities, given the possibility that this virus will be further circulating exploiting asymptomatic subjects as carriers. Finally, this emergency could serve as a turning point for re-considering measures of protection of cancer patients from infectious risks as well as for learning the lesson of prioritizing the treatments. We hope that our complex organizing strain will be of utmost help to all the oncologists that, around the world, need to carry out the defense of all the vulnerable cancer patients and guarantee the preservation of the precious health of our colleagues.

Acknowledgements We thank Maria Carla and Marco Barducci for their valuable contribution in revising the English text.

Author contributions All the listed authors have significantly contributed to the final version of this manuscript (conceptualization, writing and finalization). All authors that have substantially contributed to this manuscript were mentioned in the authorship. No funding was necessary for the realization of this manuscript as in the nature of a short communication. All submitted figures and attachments were originally designed by the authors.

Funding No funding was necessary for writing the present manuscript.

\section{Compliance with ethical standards}

Conflict of interest Garattini Silvio Ken declares that he has no conflict of interest, Bin Alessandra declares that she has no conflict of interest, Donato Raffaela declares that she has no conflict of interest, Mansutti Mauro declares that he has no conflict of interest, Rizzato Simona declares that she has no conflict of interest, Troiero Graziella declares that she has no conflict of interest, Candoni Anna declares that she has no conflict of interest, Fanin Renato declares that he has no conflict of interest, and Fasola Gianpiero declares that he has no conflict of interest.

Ethical approval This article does not contain any studies with human participants or animals performed by any of the authors.

\section{Reference}

1. Wu Z, McGoogan JM. Characteristics of and Important Lessons From the Coronavirus Disease 2019 (COVID-19) Outbreak in China: Summary of a Report of 72314 Cases From the Chinese Center for Disease Control and Prevention. JAMA. 2020;

2. A Novel Coronavirus from Patients with Pneumonia in China, 2019. - PubMed - NCBI [Internet]. [cited 2020 Apr 1]. Available from: https://www.ncbi.nlm.nih.gov/pubmed/31978945

3. Lu R, Zhao X, Li J, Niu P, Yang B, Wu H, et al. Genomic characterisation and epidemiology of 2019 novel coronavirus: implications for virus origins and receptor binding. Lancet Lond Engl. 2020;395:565-74.

4. COVID-19 ITALIA - Mobile [Internet]. [cited 2020 Apr 1]. Available from: https://opendatadpc.maps.arcgis.com/apps/opsdashboa rd/index.html\#/dae18c330e8e4093bb090ab0aa2b4892

5. ESMO. COVID-19: supporting oncology professionals [Internet]. [cited 2020 Apr 2]. Available from: https://www.esmo.org/newsr oom/covid-19-and-cancer/supporting-oncology-professionals

6. A I, C A, L C, M C, B D, G F, et al. Reorganisation of Medical Oncology Departments During the Novel Coronavirus disease-19 Pandemic: A Nationwide Italian Survey [Internet]. Eur. J. Cancer Oxf. Engl. 1990. 2020 [cited 2020 May 26]. Available from: https ://pubmed.ncbi.nlm.nih.gov/32311643/

7. Cancer care during the spread of coronavirus disease 2019 (COVID-19) in Italy: young oncologists' perspective I Esmo Open [Internet]. [cited 2020 Apr 1]. Available from: https://esmoopen. bmj.com/content/5/2/e000759

8. Hanna TP, Evans GA, Booth CM. Cancer, COVID-19 and the precautionary principle: prioritizing treatment during a global pandemic. Nat Rev Clin Oncol. 2020;1-3.

Publisher's Note Springer Nature remains neutral with regard to jurisdictional claims in published maps and institutional affiliations. 